\title{
Conditional Synthesis of an Aberrant Glycoprotein mRNA by the Internal Deletion Mutant of Vesicular Stomatitis Virus
}

\author{
RONALD C. HERMAN \\ Infectious Disease Institute, Center for Laboratories and Research, New York State Department of Health, \\ Albany, New York 12201
}

Received 18 November 1982/Accepted 4 March 1983

\begin{abstract}
The internal deletion mutant (DI-LT) derived from the heat-resistant strain of vesicular stomatitis virus synthesizes an aberrant polyadenylated mRNA (G*) containing a transcript of the partially deleted polymerase gene covalently linked to the $3^{\prime}$ end of the glycoprotein message (R. C. Herman and R. A. Lazzarini, J. Virol. 40:78-86, 1981). The heat-resistant polymerase appears to play a role in the synthesis of the abnormal G* RNA. The synthesis of $G^{*}$ correlated directly with the presence of the heat-resistant $L$ protein on the defective interfering particle template. Chimeric defective interfering particles produced by passaging DI-LT with a helper virus that encodes the wild-type vesicular stomatitis virus polymerase did not synthesize $\mathrm{G}^{*}$. The subsequent passage of the chimeric DI-LT with a heat-resistant helper virus restored the ability to synthesize the $G^{*}$ transcript. These results imply that the regulatory signals normally present at the vesicular stomatitis virus $\mathrm{G} / \mathrm{L}$ intercistronic boundary may be preserved in DI-LT. These sequences are only conditionally functional because they are recognized correctly by the wild-type but not by the heat-resistant polymerase.
\end{abstract}

Vesicular stomatitis virus (VSV), a rhabdovirus, is the prototype of the negative-strand viruses. Its genome is a single-stranded $42 \mathrm{~S}$ RNA with a molecular weight of approximately $4 \times 10^{6}$. The VSV genome is transcribed into a separate, capped, and polyadenylated [poly(A) ${ }^{+}$] mRNA for each of the five proteins $(3,4,20,21)$. These have been designated nucleocapsid protein $(\mathrm{N})$, phosphoprotein (NS), matrix protein $(M)$, glycoprotein $(G)$, and polymerase (L). The mechanism by which VSV synthesizes its messages has not been conclusively established; two models have been proposed for this process. In the first, each message is separately initiated and terminated in a sequential manner. In the second, a polycistronic precursor is synthesized and subsequently processed into the mature monocistronic messages (reviewed in reference 4). Data have been published by several laboratories to support these models $(1,2,12,14,15,27,31-33)$.

One of the defective interfering (DI) particles derived from the heat-resistant strain of VSV (VSV-HR) was generated by an internal deletion of the parental $42 \mathrm{~S}$ RNA $(7,8,11,25)$. This particle (DI-LT) contains genetic information primarily from the $3^{\prime}$ half of the VSV genome $(19,29,30)$ and retains the parental $3^{\prime}$ terminal sequence $(9,11)$. DI-LT also retains approximately 320 to 350 nucleotides of the parental $5^{\prime}$ terminal sequence (11). Based upon hybridiza- tion and electron microscopic analyses, we have previously suggested that the deletion that gave rise to DI-LT probably occurs entirely within the polymerase gene and that this particle is likely to contain up to several hundred nucleotides of L-gene sequence on each side of the deletion point (Fig. 1; 11, 13).

DI-LT is unique among VSV DI particles because of its ability to synthesize mRNAs in vivo and in vitro $(9,17)$. These mRNAs are biologically functional, and DI-LT complements VSV mutants containing temperature-sensitive lesions in the N, NS, M, and G cistrons; as expected, DI-LT does not complement $\mathrm{L}$ protein mutants because of the deletion in that gene (16). Herman and Lazzarini (13) recently showed that some stocks of DI-LT synthesize an aberrant $\operatorname{poly}(\mathrm{A})^{+}$mRNA in vivo and in vitro. This RNA contains a transcript of the partially deleted polymerase gene covalently linked to the $3^{\prime}$ end of the glycoprotein message (denoted $G^{*}$ ) (Fig. 1). Normal-size $G$ message could not be detected when the transcripts synthesized by these stocks of DI-LT in the absence of helper virus were examined by the sensitive nitrocellulose filter hybridization technique (13).

$\mathrm{G}^{*}$ appears to be synthesized by precise transcription across the $\mathrm{G} / \mathrm{L}$ intercistronic boundary and through the deletion point in the polymerase gene. It is approximately 500 to 600 nucleotides longer than $\mathrm{G}$ message and contains a $3^{\prime}$ poly(A) 


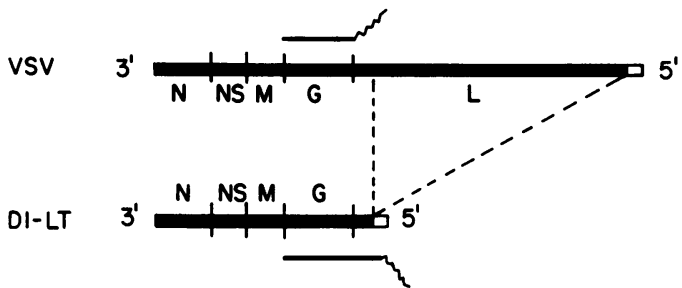

FIG. 1. Maps of VSV and DI-LT genomes. The relative lengths, as well as the locations of the intercistronic boundaries, were determined by $\operatorname{EM}(11,13$, 14). The dashed lines indicate the approximate location of the deletion in the $\mathrm{L}$ gene. The open box at the $5^{\prime}$ end of the maps represents the 320 to 350 nucleotides of the 5' parental sequence that are retained in DI-LT. The regions transcribed into the $G$ and $G^{*}$ transcripts are indicated above and below each map $(13,14)$; the wavy lines represent the poly(A) tails.

tail that is most probably encoded by the remnant L-gene polyadenylation signal (13). $\mathrm{G}^{*}$ is not detectably interrupted by an intervening $\operatorname{poly}(\mathrm{A})$ of the type that was previously described $(14,15)$. This abnormal RNA may be synthesized either because of a failure to polyadenylate the nascent chain and to terminate transcription at the 5 ' end of the $G$ cistron (model 1) or perhaps because of an inability to process an abnormal polycistronic precursor (model 2). On the basis of the hybridization and electron microscopic results, I think that it is unlikely that the deletion has simply removed the $5^{\prime}$ end of the $\mathrm{G}$ cistron.

As noted above, DI-LT was generated by VSV-HR and is routinely passaged with the aid of VSV-HR helper virus. VSV-HR was selected from wild-type VSV for its ability to survive heat treatment and to form plaques at $40^{\circ} \mathrm{C}(26)$. The HR mutation(s) has not been assigned to any of the five VSV complementation groups, but it may reside in one of the polymerase subunits (L or NS). The heat-resistant polymerase has an increased tendency to transcribe across intercistronic boundaries (unpublished data) and, consequently, it is possible that $\mathrm{G}^{*}$ is synthesized because of a defect in the VSV-HR transcriptase rather than in the template DI-LT RNA itself.

I tested this hypothesis by examining the mRNAs synthesized in vivo by a chimeric DI particle preparation that was prepared by passaging DI-LT with helper virus possessing the wild-type polymerase. After two serial passages with the alternative helper virus, the chimeric DI-LT particle synthesized the normal-size G message instead of the abnormal G* RNA. A single subsequent passage of the chimeric DI particle with VSV-HR helper virus was suffi- cient to restore its ability to synthesize the aberrant $G^{*}$ message. These results suggest that the regulatory sequences normally present at the $\mathrm{G} / \mathrm{L}$ intercistronic boundary are unaffected by the deletion that gave rise to DI-LT. These sequences, however, appear to be functionally inert when transcribed by the heat-resistant polymerase.

(A preliminary report of these observations was presented at the First Annual Meeting of the American Society for Virology, Ithaca, N.Y., 1 to 5 August 1982.)

\section{MATERIALS AND METHODS}

Cell culture. Suspension BHK-21 cells were maintained in F-13 medium (GIBCO Laboratories, Grand Island, N.Y.) supplemented with glutamine, nonessential amino acids, gentamicin, and $5 \%$ fetal calf serum at between $2 \times 10^{5}$ and $8 \times 10^{5}$ cells per ml. Monolayer BHK cells were maintained in F-11 medium supplemented as indicated above and with $0.4 \%$ glucose.

Virus stocks. DI-LT(NIH) passaged with VSVHR(NIH) helper virus was obtained from R. A. Lazzarini (National Institutes of Health, Bethesda, Md.). The passage history of this stock has been described previously (11). The DI-LT(ALB) stock was prepared by a single amplification of DI-LT(NIH) with VSVHR(NIH) helper virus at $37^{\circ} \mathrm{C}$ in suspension BHK cells in this laboratory. Purified DI-LT(URB) passaged with VSV-HR(URB) helper virus was obtained from M. E. Reichmann (University of Illinois, Urbana). The DI particles had been separated from the helper virus by successive cycles of velocity sedimentation in sucrose as previously described $(5,6)$. The chimeric DI-LT(tsG31) was constructed by Bay and Reichmann by serially passaging DI-LT(URB) twice with VSV-Glasgow/tsG 31 helper virus at $31^{\circ} \mathrm{C}$ in monolayer BHK cells (6). The resultant chimeric DI particles were purified by them as described for normal DI particles. DI-LT(HR) was prepared in this laboratory by passaging the purified DI-LT $(t s \mathrm{G} 31)$ once with VSV-HR(NIH) helper virus in monolayer BHK cells at $37^{\circ} \mathrm{C}$.

Infection conditions. All infections were carried out at $37^{\circ} \mathrm{C}$ with suspension BHK cells as previously described in detail (13). The cells were prestarved for 16 to $18 \mathrm{~h}$ in phosphate-free medium before labeling with ${ }^{32} \mathrm{P}_{\mathrm{i}}$.

Isolation and analysis of RNA. Cell fractionation, RNA extraction, and agarose-urea gel electrophoresis procedures were performed exactly as described previously (13).

Nitrocellulose filter hybridizations. Blot hybridizations 'were performed as described by Thomas (34) as modified by Herman and Lazzarini (13). Recombinant plasmids pG65, pM32, and pNS173 used as probes were obtained from J. K. Rose and have been described in detail by him (28). To quantitate the relative amounts of $\mathrm{G}^{*}$ and $\mathrm{G}$ synthesized, the autoradiogram was used as a template to excise the appropriate bands from the nitrocellulose filter, and these were counted in a liquid scintillation counter with $15 \mathrm{ml}$ of Aquasol (New England Nuclear Corp., Boston, Mass.). 


\section{RESULTS}

Identification of an NS-M transcript. In a previous report, Herman and Lazzarini (13) showed that DI-LT synthesizes the aberrant $\operatorname{poly}(\mathrm{A})^{+}$ G* mRNA. This RNA contains covalently linked sequences that are transcribed from the adjacent $G$ and remnant $L$ cistrons. This abnormal RNA is fairly abundant. In a typical experiment, the ratio of ${ }^{32} \mathrm{P}$ counts per minute in the G* RNA relative to $\mathrm{N}$ message synthesized by DI-LT was 0.34 . In a parallel culture, the ratio of counts per minute in $\mathrm{G}$ message relative to $\mathrm{N}$ message synthesized by VSV-HR was 0.48 (however, see below).

Using a probe that was specific for the $3^{\prime}$ end of the VSV G message, I showed that the DILT(NIH) and DI-LT(ALB) stocks (see above for details) do not synthesize the normal-size $G$ message in the absence of helper virus. Nevertheless, these stocks can synthesize a poly(A) ${ }^{+}$ RNA which approximately comigrates with $G$ message in an agarose-urea gel (13). This RNA cannot contain $\mathbf{G}$ message sequences because it does not anneal to the G-specific probe. On the basis of electron microscopic measurements, I estimated that a complete transcript of the NS and $M$ cistrons would be approximately equal in length to $G$ message and could comigrate with it in an agarose gel. To test this, I annealed a ${ }^{32} \mathrm{P}$ labeled $\mathrm{M}$ message-specific probe to a nitrocellulose filter blot of an agarose gel containing the poly (A) ${ }^{+}$VSV-HR and DI-LT(ALB) messages.

The specificity of the probe was demonstrated by its hybridization to the $M$ messages synthesized by both VSV-HR and DI-LT(ALB) (Fig. 2 ). This probe also annealed to material that migrated to the position expected for the $G$ message. A similar result was obtained by hybridizing an NS-specific probe to the same filter (data not shown). These results showed that DILT synthesizes a poly(A) ${ }^{+}$RNA containing both NS and $M$ message sequences. Because the probe also annealed to the band containing the VSV-HR G message (Fig. 2), these observations indicate that the synthesis of NS-M was not limited to DI-LT. However, the synthesis of NSM by VSV-HR was normally masked because it comigrated with the $G$ message. Preliminary experiments suggested that the $\operatorname{poly}(\mathrm{A})$ was probably located at the $3^{\prime}$ end of NS-M (data not shown).

mRNA synthesis by chimeric DI-LT particles. To examine the influence of the heat-resistant polymerase on the synthesis of the DI-LT mRNAs, I attempted to construct a chimeric DI particle by passaging DI-LT(ALB) with the Mudd-Summers isolate of VSV-Indiana (23) in suspension BHK cells. Since DI-LT cannot synthesize the $\mathrm{L}$ protein, the resulting DI particles

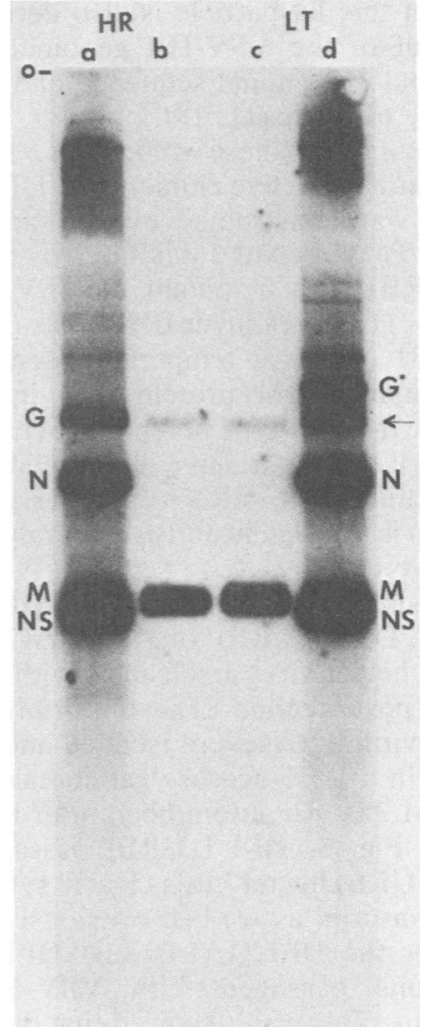

FIG. 2. Nitrocellulose filter hybridization with plasmid pM32. BHK cells were infected in the presence of actinomycin $\mathrm{D}$ and labeled with $\left[5-{ }^{3} \mathrm{H}\right]$ uridine $(25 \mu \mathrm{Ci} / \mathrm{ml})$ or ${ }^{32} \mathrm{P}_{\mathrm{i}}(100 \mu \mathrm{Ci} / \mathrm{ml})$. The RNA was extracted with phenol-chloroform, and $\operatorname{poly}(\mathrm{A})^{+}$sequences were selected by oligodeoxythymidylatecellulose chromatography. Equal amounts of the ${ }^{3} \mathrm{H}$ labeled mRNA were resolved in a $1.5 \%$ agarose-urea gel for $18 \mathrm{~h}$ at $120 \mathrm{~V}$ and then transferred to a nitrocellulose filter by blotting $(13,34)$. The filter was hybridized with ${ }^{32} \mathrm{P}$-labeled plasmid pM32 (28) and exposed against Kodak XAR film with an intensifying screen at $-70^{\circ} \mathrm{C}$. Lane a, ${ }^{32} \mathrm{P}$-labeled VSV-HR marker RNA; lane b, ${ }^{3} \mathrm{H}$-labeled VSV-HR RNA; lane c, ${ }^{3} \mathrm{H}-$ labeled DI-LT(NIH) RNA; lane d, ${ }^{32}$ P-labeled DILT(NIH) mRNA. The arrow points to the NS-M band.

should contain only those polymerase molecules that are encoded by the helper virus. These particles could, of course, contain mixtures of the other four VSV proteins that are synthesized by both DI-LT and the helper virus. Unfortunately, after two serial passages with VSV-Indiana, the resulting purified DI particle preparation no longer retained the capacity to synthesize mRNA in vivo. This probably occurred because of the preferential replication of another DI particle known to contaminate most, if not all, DI-LT stocks (11). Although the 
genome of this DI particle is also derived from the $3^{\prime}$ half of the VSV-HR genome, it has a nonparental 3' terminal sequence and does not synthesize mRNAs $(11,18)$.

To circumvent these difficulties, I used a transcriptionally active chimeric DI-LT preparation that was constructed by Bay and Reichmann (6). They prepared this stock by passaging DI-LT(URB) with a mutant of VSV-Glasgow (VSV-tsG31) in monolayer BHK cells. Although VSV-tsG31 carries a temperature-sensitive lesion in the matrix $(\mathrm{M})$ protein, this virus should synthesize the wild-type polymerase $(\mathrm{L})$ protein. Bay and Reichmann have confirmed this by demonstrating that mRNA synthesis by DI$\mathrm{LT}(t s \mathrm{G} 31)$ is not significantly temperature sensitive in vivo (6).

Suspension BHK cells were infected with DILT(ALB), DI-LT(URB), or DI-LT( $t s$ G31) without added helper virus and labeled with ${ }^{32} \mathrm{P}_{i}$ from 1 to $4 \mathrm{~h}$ postinfection. The uniformly labeled $\operatorname{poly}(\mathrm{A})^{+}$viral RNAs were isolated and electrophoresed in a $1.5 \%$ agarose gel containing $6 \mathrm{M}$ urea at $\mathrm{pH}$ 3.0. An autoradiogram of this gel is shown in Fig. 3. DI-LT(URB) passaged with VSV-HR(URB) helper virus clearly synthesized the $\mathrm{G}^{*}$ transcript as we had previously demonstrated for the DI-LT(ALB) and DI-LT(NIH) preparations passaged with VSV-HR(NIH) helper virus. This result showed that the synthesis of $\mathrm{G}^{*}$ was not unique to the closely related DI-LT(ALB) and DI-LT(NIH) stocks and did not depend on a single stock of VSV-HR helper virus. The intensity of the $G^{*}$ band relative to the one migrating ahead of it appeared, however, to be somewhat different for the DILT(ALB) and DI-LT(URB) stocks (see below).

After two serial passages with VSV-tsG31 helper virus, the purified chimeric DI-LT $(t s G 31)$ no longer synthesized the $G^{*}$ transcript. Only a single major band that migrated to the position expected for G message and NS-M was visible in the appropriate region of the gel (Fig. 2, lane c). No other effects on the mobility of the mRNAs synthesized by DI-LT( $t s \mathrm{G} 31)$ were observed. Taken together, these observations showed that the structure of a DI-LT transcription product was directly affected by the helper virus used to propagate the DI particles and suggested that the synthesis of $G^{*}$ required the presence of the heat-resistant polymerase.

Resumption of $\mathbf{G}^{*}$ synthesis. To demonstrate that the failure to synthesize the $\mathrm{G}^{*}$ transcript was due solely to the absence of the heatresistant polymerase, I passaged the purified chimeric DI-LT(tsG31) particles once with VSV-HR(NIH) helper virus to reintroduce the heat-resistant polymerase onto the DI-LT template. Suspension BHK cells were infected with the resulting DI-LT(HR) particles and labeled

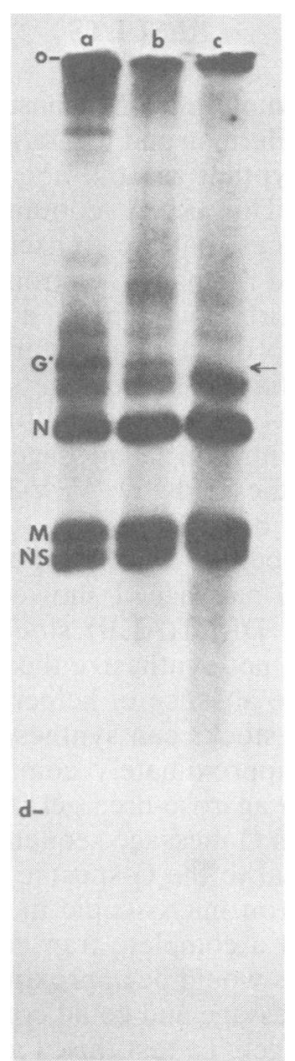

FIG. 3. Poly(A) ${ }^{+}$mRNA synthesized by the chimeric DI particle. BHK cells were infected as outlined in the legend to Fig. 2. The mRNA was labeled with ${ }^{32} \mathrm{P}_{\mathrm{i}}$ for $3 \mathrm{~h}$. The poly $(\mathrm{A})^{+}$RNA was isolated and electrophoresed in an agarose-urea gel for $18 \mathrm{~h}$ at $90 \mathrm{~V}$. Lane a, DI-LT(ALB); lane b, DI-LT(URB); lane c, chimeric DI-LT $(t s \mathrm{G} 31), o$ and $d$ indicate the origin and location, respectively, of the bromophenol blue tracking dye. Note the absence of $G^{*}$ in lane $c$ (arrow).

with ${ }^{32} \mathrm{P}_{\mathrm{i}}$. The poly $(\mathrm{A})^{+}$RNAs were electrophoresed in an agarose-urea gel in parallel with the labeled messages synthesized by DI-LT(URB) and DI-LT( $t s \mathrm{G} 31)$.

After only a single passage of DI-LT(tsG31) with VSV-HR(NIH) helper virus, the resulting DI-LT(HR) particles regained the ability to synthesize the $G^{*}$ transcript in vivo (Fig. 4). These observations showed that the synthesis of $\mathrm{G}^{*}$ by DI-LT was determined by the helper virus and that the synthesis of $\mathrm{G}^{*}$ could be directly correlated with the presence of the heat-resistant $L$ protein. These findings imply that the correct regulatory sequences are probably present at the $\mathrm{G} / \mathrm{L}$ intercistronic boundary in the DI-LT genome even though they are recognized by the VSV-Glasgow polymerase and not by the VSVHR enzyme. 


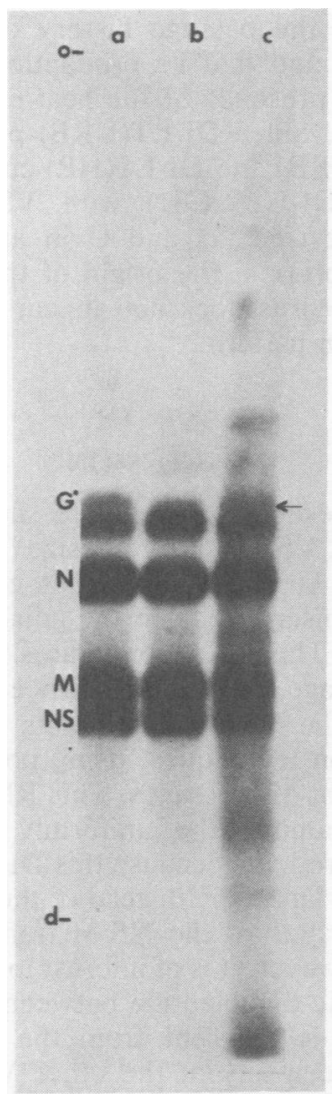

FIG. 4. Resumption of $\mathrm{G}^{*}$ synthesis. Uniformly ${ }^{32} \mathrm{P}$-labeled poly $(\mathrm{A})^{+}$mRNA was prepared as outlined in the legends to Fig. 2 and 3. Lane a, DI-LT(URB); lane b, DI-LT(tsG31); lane c, DI-LT(HR). The symbols are explained in the legend to Fig. 3. The arrow points to the $\mathrm{G}^{*}$ band synthesized by DI-LT(HR).

Nitrocellulose filter hybridizations. As indicated above, the relative mobility of a VSV product in an agarose-urea gel may not necessarily prove its sequence identity. Therefore, to confirm the results obtained with the chimeric DI particles, I repeated this series of experiments, using nitrocellulose filter hybridization techniques with a probe that was specific for the $G$ message. The infections were performed exactly as outlined above, except that the virus-specific RNAs were labeled with $\left[{ }^{3} \mathrm{H}\right]$ uridine instead of ${ }^{32} \mathrm{P}_{\mathrm{i}}$. After electrophoresis in an agarose-urea gel, the poly $(A)^{+}$RNAs were transferred to a nitrocellulose filter by blotting and were subsequently hybridized with a ${ }^{32} \mathrm{P}$-labeled $\mathrm{G}$ message probe. The results of this experiment are shown in Fig. 5.

The predominant DI-LT(ALB) RNA detected by the $G$ probe migrated to the position expected for the $G^{*}$ transcript. A relatively small amount of normal-size $\mathbf{G}$ message was also detected by the probe. This was probably synthesized by a low level of residual VSV-HR contaminating this particular preparation of DI-LT(ALB). A faint band of $31 \mathrm{~S} \mathrm{~L}$ message is perhaps visible in Fig. 5, lane e, containing the uniformly labeled DI-LT(ALB) mRNA, and this confirms the presence of a small number of infectious virions in this preparation.

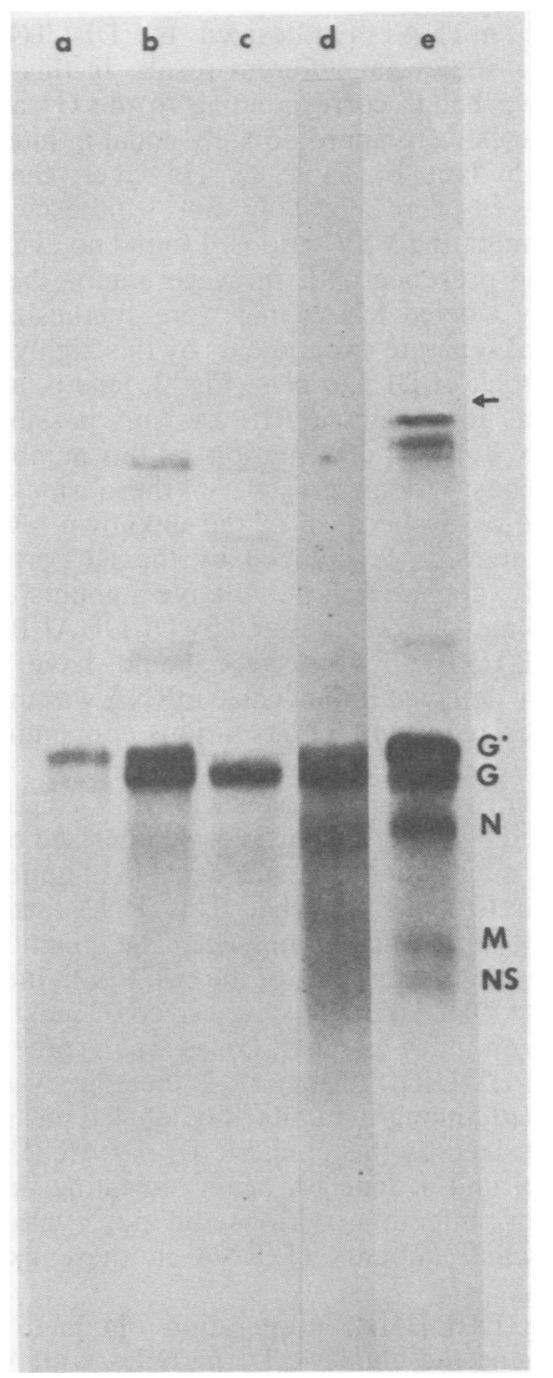

FIG. 5. Nitrocellulose filter hybridization. ${ }^{3} \mathrm{H}$-labeled poly $(\mathrm{A})^{+}$mRNA was isolated and resolved in an agarose-urea gel. The RNAs were transferred to nitrocellulose by blotting. The VSV-specific sequence was excised and purified from plasmid pG65 (28) before nick translation. Lane a, DI-LT(ALB); lane b, DILT(URB); lane c, DI-LT( $t s$ G31); lane d, DI-LT(HR); lane e, uniformly ${ }^{32}$ P-labeled DI-LT(ALB) marker mRNA. The positions of $G^{*}$ and $G$, as well as the other small messages, are indicated at the right. The arrow points to the faint band of $\mathrm{L}$ mRNA present in lane $e$. 
TABLE 1. Synthesis of $G^{*}$ and $G$ messages

\begin{tabular}{lccc}
\hline \multirow{2}{*}{ Stock } & \multicolumn{3}{c}{ Transcript } \\
\cline { 2 - 4 } & $\begin{array}{c}\mathrm{G}^{*} \\
(\mathrm{cpm})^{a}\end{array}$ & $\begin{array}{c}\mathrm{G} \\
(\mathrm{cpm})\end{array}$ & $\mathrm{G}^{*} / \mathrm{G}$ \\
\hline DI-LT(URB) & 536 & 803 & 0.67 \\
DI-LT(tsG31) & 105 & 756 & 0.14 \\
DI-LT(HR) & 64 & 113 & 0.57 \\
\hline
\end{tabular}

${ }^{a}$ Radioactivity bound to nitrocellulose filter bands.

The mRNAs synthesized by DI-LT(URB) gave a somewhat different result. In this case, the two bands corresponding to the $G^{*}$ and $G$ messages were approximately equal in intensity (Fig. 5, lane b; Table 1). However, these G messages were probably not synthesized by contaminating VSV virions. I found no evidence for the presence of $L$ message among the uniformly labeled RNAs that were synthesized in several separate experiments by this highly purified stock of DI particles (Fig. 3, lane b, and 4, lane $a$, for example). It was not possible to obtain a direct quantitation of the number of infectious virions present in these stocks by plaque assay because of the unknown level of auto-interference induced by the DI particles. Clearly, considering the relative amounts of $\mathrm{G}^{*}$ and $G$ message synthesized by DI-LT(ALB) and DI-LT(URB), L message should have been readily detected if the G-size mRNA was merely synthesized by infectious virions contaminating the latter stock.

After two serial passages with VSV-tsG31, the purified chimeric DI particles synthesized only a single major band that was capable of annealing to the G-specific probe. This RNA had the mobility of normal $\mathrm{G}$ message. The quantitation of the relative amounts of the two RNAs indicated that DI-LT(tsG31) synthesized at most only about one fifth as much $\mathrm{G}^{*}$ as was synthesized by DI-LT(URB) (Table 1). L message was not detected among the uniformly labeled messages synthesized by this chimeric DI particle (Fig. 3, lane $c$, and 4, lane b), again indicating that, if present, infectious virions did not synthesize significant amounts of RNA in these experiments.

The DI-LT(HR) preparation obtained after passaging the chimeric DI particles with VSVHR(NIH) helper virus synthesized two RNAs that annealed to the $G$ probe. These corresponded to $G^{*}$ and $G$ messages. $G^{*}$ and $G$ were present in approximately the same relative proportions as were the two similar species synthesized by the original DI-LT(URB) stock (Fig. 5, lane $c$, and Table 1). The results obtained with the G-specific probe confirm and extend those obtained with the uniformly labeled mRNA species. They indicate that the synthesis of $G^{*}$ varies with the passage history of the DI-LT preparation and that its production appears to require the presence of the heat-resistant VSV polymerase. Since DI-LT(URB) passaged with VSV-HR(URB) and DI-LT(HR) constructed by passaging DI-LT $(t s \mathrm{G} 31)$ with VSV-HR(NIH) both synthesized $G^{*}$ and $G$ in approximately equal proportions, the origin of the heat-resistant helper virus does not appear to affect the transcription pattern.

\section{DISCUSSION}

I have presented evidence to show that both DI-LT and VSV-HR synthesize a previously undetected, large poly(A) ${ }^{+}$RNA containing sequences transcribed from the adjacent NS and $M$ cistrons. This RNA comigrates with the normal $\mathrm{G}$ message and consequently can be detected among the VSV-HR products only by blot hybridization techniques, using probes specific for the NS or M messages. This RNA is readily detectable among the uniformly labeled DILT(ALB) products because this DI particle synthesizes the larger $G^{*}$ instead of the $G$ message. The significance of the NS-M transcript is not known. However, it is of interest to note that the intercistronic dinucleotide between the NS and $M$ cistrons is different from the dinucleotide found between any of the other VSV cistrons $(20,28)$. This junction is also the site at which the VSV transcriptase most frequently synthesizes an intervening poly (A) in vitro (12). Preliminary results suggest that the Mudd-Summers isolate of VSV-Indiana may also synthesize this transcript (unpublished data).

The results obtained with the chimeric DI-LT particles demonstrate that the synthesis of the $\mathrm{G}^{*}$ transcript can be directly correlated with the presence of the heat-resistant polymerase. DILT(URB) synthesizes the aberrant $G^{*}$ transcript when passaged with VSV-HR(URB) helper virus but synthesizes only the normal-size $\mathrm{G}$ message when passaged with VSV-tsG31. These findings imply that the regulatory sequences normally present at the $\mathrm{G} / \mathrm{L}$ intercistronic boundary may be preserved in the DI-LT genome. These sequences appear, however, to be only conditionally functional. They are biologically inert when encountered by the VSVHR polymerase, but they can, nevertheless, program the VSV-Glasgow polymerase correctly. The apparent preservation of a functional $\mathrm{G} / \mathrm{L}$ intercistronic junction in this DI particle is consistent with the earlier suggestion that the deletion which gave rise to DI-LT probably occurred entirely within the polymerase gene $(11,13)$.

The reason for the differential recognition of 
these sequences by the VSV-HR and VSVGlasgow polymerases is not known, and a number of models could be envisioned to explain this phenomenon. The validity of any one of these models depends ultimately on the identity of the protein(s) that is directly responsible for this effect and on the precise nature of the transcription mechanism itself. Regardless of the exact cause, these results show that the VSV-HR transcriptase can transcribe across what appears to be an intact and potentially functional intercistronic boundary in vivo. The product of this read-through phenomenon is an abundant and biologically active mRNA (Liberman and Herman, unpublished data).

The interpretation of these results is somewhat complicated by the observation that DILT(URB) synthesized both $G^{*}$ and $G$ in approximately equal proportions, whereas DI-LT(NIH) and DI-LT(ALB) synthesized only $\mathrm{G}^{*}$ in the absence of a helper virus. It is unlikely that the synthesis of $G$ message by the DI-LT(URB) stock was directed only by contaminating standard virions since this stock was purified by several cycles of velocity sedimentation in sucrose. Under these conditions, Bay and Reichmann estimate that perhaps only one infectious virion per $10^{4}$ biologically active DI particles remains (5). This conclusion is substantiated by the observation that $\mathrm{L}$ message was not detected among the uniformly labeled transcripts synthesized in vivo by either DI-LT(URB) or DILT $(t s \mathrm{G} 31)$. On the other hand, even though the preparation of DI-LT(ALB) used in these experiments synthesized only a very small amount of $G$ message relative to that of $G^{*}$, some $L$ message was detected among its uniformly labeled messages. It is likely that this $\mathrm{G}$ message was synthesized by contaminating infectious VSV.

Perhaps the simplest explanation of these results is that the DI-LT(URB) stock contains two DI particles that retain the ability to synthesize mRNA. One of these may synthesize G message, and the other may synthesize $G^{*}$. The passage histories of DI-LT(ALB) and DILT(URB) are significantly different, and it is possible that another DI particle containing genetic information from the $3^{\prime}$ half of the VSVHR genome arose during propagation. The DILT(NIH) seed stock, from which DI-LT(ALB) was grown, is only one passage beyond the original stock of DI-LT that was prepared by Kang (11). The DI-LT(NIH) and DI-LT(ALB) stocks synthesize only the $G^{*}$ transcript in the absence of a helper virus, and they are likely to contain the original "DI-LT" particle. The confirmation of these conclusions will require the biological cloning of the DI-LT(URB) stock and the subsequent analysis of the mRNAs synthe- sized by the transcriptionally active DI particles selected from it.

Because two DI particles capable of synthesizing mRNA may be present in the DILT(URB) stock, the possibility remains that these results only reflect the selective amplification of different DI particles by the alternative helper viruses. This explanation cannot be ruled out by the data presented here. Such an interpretation would require that the DI particles synthesizing normal $G$ message have approximately a twofold replicative advantage over the particles synthesizing $\mathrm{G}^{*}$ during each passage with VSV$t s \mathrm{G} 31$. At the same time, the particles synthesizing $\mathrm{G}^{*}$ would require approximately a fourfold advantage during passage with VSV-HR to explain the data presented in Table 1.

It is relevant to note here that Perrault et al. (24) have also observed an effect of the helper virus on RNA synthesis. They have isolated a strain of VSV (VSV-pol R1) that has an increased tendency to transcribe across intercistronic boundaries. DI particles passaged with the aid of VSV-pol R1 virus synthesize almost complete copies of the DI particle genome in vitro, whereas these same DI particles synthesize only a 46-nucleotide-long product when passaged with wild-type helper virus. Although VSV-pol R1 is not heat resistant, both it and VSV-HR were isolated after multiple cycles of heat inactivation of the wild-type virus $(24,26)$.

At the present time, the identity of the protein that is responsible for the differential transcription is not known. We may be able to exploit this difference to identify the protein(s) causing this phenomenon. In vitro reconstitution experiments have been shown to be useful for assessing the role of the individual VSV proteins in viral transcription (10). Various combinations of $\mathrm{L}$ and NS isolated from infectious VSV-HR and VSV-Glasgow virions can be reassembled onto stripped DI-LT nucleocapsids, and the synthesis of $G^{*}$ and $G$ can be monitored in vitro. Since $L$ protein is the only VSV polypeptide that can be completely replaced by passaging DI-LT with an alternative helper virus, it is not unreasonable to propose that the $\mathrm{L}$ protein is responsible for these effects.

None of the DI-LT stocks used in these experiments synthesized detectable amounts of a small poly $(A)^{+}$" $L^{*}$ "' RNA transcribed from the remnant polymerase gene. The $\mathrm{L}^{*}$ RNA could potentially contain approximately 300 to 400 nucleotides of $\mathrm{L}$ message sequence plus a poly(A) tail. Thus, even though the chimeric DILT( $t s G 31)$ synthesized only the normal-size G message, I found no evidence to indicate that it could also synthesize $L^{*}$ either by reinitiating transcription at the $3^{\prime}$ end of the remnant $L$ gene or by cleaving the polycistronic $\mathrm{G}^{*}$ message. 
This assumes that such an RNA would be metabolically stable and synthesized in a quantity sufficient to be detected by uniformly labeling the messages with ${ }^{32} \mathrm{P}_{\mathrm{i}}$. Experiments are in progress to reexamine this question with the use of a probe that is specific for the $5^{\prime}$ end of the $L$ message.

In summary, the data presented here describe a unique situation, in which a sequence assumed to play a role in the normal regulation of VSV mRNA synthesis appears to be only conditionally functional. The wild-type VSV-Glasgow polymerase recognizes this sequence, whereas the VSV-HR polymerase does not. The VSV-HR polymerase seems quite capable of interpreting all of the other intercistronic junction sequences correctly since DI-LT passaged with VSV-HR helper virus does not synthesize a spectrum of polycistronic transcripts. The one possible exception may be the NS-M RNA. However, the $\mathrm{NS} / \mathrm{M}$ boundary is distinctive from the other junctions, and unusual transcription phenomena are known to occur in this region $(12,28)$. Consequently, additional and as yet undefined factors may also play a role in the regulation of VSV mRNA biosynthesis.

\section{ACKNOWLEDGMENTS}

I am indebted to M. E. Reichmann for generously providing the chimeric DI particle stocks used in these experiments. I also thank R. A. Lazzarini for providing DI-LT(NIH), J. K. Rose for providing the cloned VSV sequences, and G. P. Johnson for his expert technical assistance.

This work was supported by Public Health Service grant AI17699 from the National Institute of Allergy and Infectious Diseases.

\section{LITERATURE CITED}

1. Ball, L. A. 1977. Transcriptional mapping of vesicular stomatitis virus in vivo. J. Virol. 21:4111-414.

2. Ball, L. A., and C. N. White. 1976. Order of transcription of genes of vesicular stomatitis virus. Proc. Natl. Acad. Sci. U.S.A. 73:442-446.

3. Baltimore, D., A. S. Huang, and M. Stampfer. 1970. Ribonucleic acid synthesis of vesicular stomatitis virus. II. An RNA polymerase in the virion. Proc. Natl. Acad. Sci. U.S.A. 66:572-576.

4. Banerjee, A. K., G. Abraham, and R. J. Colonno. 1977. Vesicular stomatitis virus: mode of transcription. J. Gen. Virol. 34:1-8.

5. Bay, P. H. S., and M. E. Reichmann. 1979. UV inactivation of the biological activity of defective interfering particles generated by vesicular stomatitis virus. J. Virol. 32:876-884.

6. Bay, P. H. S., and M. E. Reichmann. 1982. In vitro and in vivo inhibition of primary transcription of vesicular stomatitis virus by a defective interfering particle. J. Virol. 41:172-182.

7. Chanda, P. K., C. Y. Kang, and A. K. Banerjee. 1980. Synthesis in vitro of the full-length complement of defective-interfering particle RNR of vesicular stomatitis virus. Proc. Natl. Acad. Sci. U.S.A. 77:3927-3931.

8. Clerx-Van Haaster, C., J. P. Clewley, and D. H. L. Bishop. 1980. Oligonucleotide sequence analyses indicate that vesicular stomatitis virus large defective interfering parti- cle RNA is made by internal deletion: evidence for similar transcription polyadenylation signals for the synthesis of all vesicular stomatitis virus mRNA species. J. Virol. 33:807-817.

9. Colonno, R. J., R. A. Lazzarini, J. D. Keene, and A. K. Banerjee. 1977. In vitro RNA synthesis of messenger RNA by a defective interfering particle of vesicular stomatitis virus. Proc. Natl. Acad. Sci. U.S.A. 74:1884-1888.

10. Emerson, S. U., and Y. H. Yu. 1975. Both NS and L are required for in vitro RNA synthesis by vesicular stomatitis virus. J. Virol. 15:1348-1356.

11. Epstein, D. L., R. C. Herman, I. M. Chien, and R. A. Lazzarini. 1980. Defective interfering particle generated by internal deletion of the vesicular stomatitis virus genome. J. Virol. 33:818-829.

12. Herman, R. C., S. Adler, R. A. Lazzarini, R. J. Colonno, A. K. Banerjee, and H. Westphal. 1978. Intervening polyadenylate sequences in RNA transcripts of vesicular stomatitis virus. Cell 15:587-596.

13. Herman, R. C., and R. A. Lazzarini. 1981. Aberrant glycoprotein mRNA synthesized by the internal deletion mutant of vesicular stomatitis virus. J. Virol. 40:78-86.

14. Herman, R. C., and R. A. Lazzarini. 1981. Vesicular stomatitis virus RNA polymerase can read through the boundary between the leader and $\mathrm{N}$ genes in vitro. $\mathrm{J}$. Virol. 38:792-796.

15. Herman, R. C., M. Schubert, J. D. Keene, and R. A. Lazzarini. 1980. Polycistronic vesicular stomatitis virus RNA transcripts. Proc. Natl. Acad. Sci. U.S.A. 77:46624665.

16. Johnson, L. D., M. Binder, and R. A. Lazzarini. 1979. A defective interfering vesicular stomatitis virus particle that directs the synthesis of functional proteins in the absence of helper virus. Virology 99:203-206.

17. Johnson, L. D., and R. A. Lazzarini. 1977. Replication of a viral RNA by a defective interfering vesicular stomatitis virus particle in the absence of helper virus. Proc. Natl. Acad. Sci. U.S.A. 74:4387-4391.

18. Keene, J. D., I. M. Chien, and R. A. Lazzarini. 1981. Vesicular stomatitis virus defective interfering particle containing a muted internal leader RNA gene. Proc. Natl. Acad. Sci. U.S.A. 78:2090-2094.

19. Leamnson, R. D., and M. E. Reichmann. 1974. The RNA of defective vesicular stomatitis virus particles in relation to viral cistrons. J. Mol. Biol. 85:551-568.

20. McGeoch, D. J. 1979. Structure of the gene N: gene NS intercistronic junction in the genome of vesicular stomatitis virus. Cell 17:637-681.

21. Moyer, S. A., and A. K. Banerjee. 1975. Messenger RNA species synthesized in vitro by the virion-associated RNA polymerase of vesicular stomatitis virus. Cell 4:37-43.

22. Moyer, S. A., M. J. Grubman, E. Ehrenfeld, and A. K. Banerjee. 1975. Studies on the in vivo and the in vitro messenger RNA species of vesicular stomatitis virus. Virology 67:463-473.

23. Mudd, J. A., and D. F. Summers. 1970. Protein synthesis in vesicular stomatitis virus-infected HeLa cells. Virology 42:328-340.

24. Perrault, J., J. L. Lane, and M. A. McClure. 1980. A variant of VSV generates defective interfering particles with replicase-like activity in vitro, p. 379-390. In B. Fields, R. Jaenis, and C. F. Fox (ed.), Animal virus genetics. ICN-UCLA Symposium on Molecular and Cellular Biology, vol. XVIII. Academic Press, Inc., New York.

25. Perrault, J., and B. Semler. 1979. Internal deletions in two distinct classes of defective interfering particles of vesicular stomatitis virus. Proc. Natl. Acad. Sci. U.S.A. 76:6191-6195.

26. Petric, M., and L. Prevec. 1970. Vesicular stomatitis virus-a new interfering particle, intracellular structures, and virus-specific RNA. Virology 41:615-630.

27. Rose, J. K. 1975. Heterogeneous 5'-terminal structures occur on vesicular stomatitis virus mRNAs. J. Biol. Chem. 250:8098-8104. 
28. Rose, J. K. 1980. Complete intergenic and flanking sequences from the genome of vesicular stomatitis virus. Cell 19:415-421.

29. Schnitzlein, W. M., and M. E. Reichmann. 1976. The size and the cistronic origin of defective interfering virus particle RNAs in relation to homotypic and heterotypic interference. J. Mol. Biol. 101:307-325.

30. Stamminger, G., and R. A. Lazzarini. 1974. Analysis of the RNA of defective VSV particles. Cell 3:85-93.

31. Testa, D., and A. K. Banerjee. 1978. In vitro synthesis of a possible precursor RNA by purified vesicular stomatitis virus. Biochem. Biophys. Res. Commun. 82:655-664.

32. Testa, D., P. K. Chanda, and A. K. Banerjee. 1980. In vitro synthesis of the full-length complement of the negative-strand genome RNA of vesicular stomatitis virus. Proc. Natl. Acad. Sci. U.S.A. 77:294-298.

33. Testa, D., P. K. Chanda, and A. K. Banerjee. 1980. Unique mode of transcription in vitro by vesicular stomatitis virus. Cell 20:267-275.

34. Thomas, P. S. 1980. Hybridization of denatured RNA and small DNA fragments transferred to nitrocellulose. Proc. Natl. Sci. U.S.A. 77:5201-5205. 